Current cancer radiotherapy relies on increasingly high dose rates of ionising radiation (100-2400 cGy/min). It is possible that changing dose rates is not paralleled by treatment effectiveness.

Irradiating cancer cells is assumed to induce molecular alterations that ultimately lead to apoptotic death. Studies comparing the efficacy of radiation-induced DNA damage and apoptotic death in relation to varying dose rates do not provide unequivocal data. Whereas some have demonstrated higher dose rates (single dose) to effectively kill cancer cells, others claim the opposite. Recent gene expression studies in cells subject to variable dose rates stress alterations in molecular signalling, especially in the expression of genes linked to cell survival, immune response, and tumour progression. Novel irradiation techniques of modern cancer treatment do not rely anymore on maintaining absolute constancy of dose rates during radiation emission: instead, timing and exposure areas are regulated temporally and spatially by modulating the dose rate and beam shape. Such conditions may be reflected in tumour cells' response to irradiation, and this is supported by the references provided.

Key words: cancer radiotherapy, ionising radiation, dose rate, apoptosis, genetic damage, gene expression profile.

Contemp Oncol (Pozn) 2016; 20 (1): 449-452 DOI: https://doi.org/10.5114/wo.2016.65603

\section{Can high dose rates used in cancer radiotherapy change therapeutic effectiveness?}

\author{
Maria Konopacka ${ }^{1}$, Jacek Rogoliński ${ }^{1}$, Aleksander Sochanik ${ }^{1}$, \\ Krzysztof Ślosarek²
}

${ }^{1}$ Centre for Translational Research and Molecular Biology of Cancer, Maria Skłodowska-Curie Memorial Cancer Centre and Institute of Oncology Gliwice Branch, Gliwice, Poland ${ }^{2}$ Department of Radiotherapy and Brachytherapy Planning, Maria Skłodowska-Curie Memorial Cancer Centre and Institute of Oncology Gliwice Branch, Gliwice, Poland

\section{Dose rate and biological effects in irradiated cells}

Biological effects of irradiation such as DNA damage, induction of mutations and cell death all depend on the kind, dose, and conditions of irradiation (dose rate and fractionation as well as origin, mass and oxygenation status of the irradiated tissue).

The radiation dose rate $(P)$ is defined as the dose absorbed within unit of time:

$$
P=d D / d t[G y / s]
$$

where $\mathrm{D}$ is energy absorbed.

A predefined radiation dose can be delivered by employing variable timespans and modulating dose rates. Studies of dose rate impact on the extent of radiation-induced biological effects have a long track record. The most clear-cut effects related to magnitude of dose rates were reported for the 1-100 cGy/min range [1]. For this particular dose rate span an inverse effect was described: small dose rates are more effective in inducing genetic damage and cell death as compared to high dose rates for the same magnitude of radiation dose [2-6]. Until recently dose rate changes exceeding 100 cGy/ min were not thought to exert any special effect on cancer cells' response to radiotherapy. It has been commonly assumed that biological properties affecting cells subjected to low LET irradiation depend on dose rate whereas in cells exposed to high LET irradiation such effects are independent of this parameter [7].

\section{Effect of dose rate on cell survival}

It has long been believed that low LET radiation such as that used in cancer radiotherapy obtained from cobalt sources and linear accelerators is more effective in cancer cell killing when used at high dose rates [1]. Based on newer results generated using modern irradiation techniques that aim to achieve higher and higher dose rates, a trend of decreased cell survival at higher dose rates has been emerging. Theoretical considerations and results generated in vitro by Keall et al. demonstrated that longer exposure times (thus, decreased dose rates) are a substantial factor enhancing survival of irradiated cells [8]. Other investigators have shown that the percentage of cancer cells surviving irradiation using identical doses is higher in the case of 5-minute exposure compared to survival following 1-minute delivery [9]. This is in agreement with the results of experiments performed using cultured human glioma cells the survival of which decreased more effectively following higher dose rate of irradiation (400-2400 cGy/min range) [10, 
11]. However, other researchers who employed a similar dose rate range, found no differences in survival of different cell lines investigated. Sorenson et al. did not observe any survival effect of 500-3000 cGy/min dose rates in the case of V79 (Chinese hamster lung fibroblasts) and FaDu (human head and neck carcinoma) cell lines [12]. It was also demonstrated that $600 \mathrm{cGy} / \mathrm{min}$ dose rate irradiation, as compared to $2400 \mathrm{cGy} / \mathrm{min}$, did not change survival in three human cancer cell lines (astrocytes, glioma and lung cancer cells) [13]. The differences in cell survival observed in these reports could arise from cell lines' specificity as well as irradiation conditions.

\section{Effect of dose rate on DNA damage}

It has been widely accepted that single- and doublestrand breaks are among the most important types of DNA damage. Histon $\gamma$-H2AX is considered to be a reliable marker of double-stranded DNA breaks that form in irradiated cells [14]. Another established biomarker of radiation exposure is micronuclei formation in dividing cultured cells [15].

The greater impact of higher dose rates has been confirmed in a study report concerning mice irradiated with two different dose rates ( $0.31 \mathrm{cGy} / \mathrm{min}$ and 1.03 cGy/min) [16]. Cells containing micronuclei and apoptotic nuclei, as well as H2AX histone, were detected among peripheral blood lymphocytes. No effect of dose rate on the induction of apoptosis and micronuclei was found, but the level of H2AX, which reflects the number of DNA strand breaks was higher in lymphocytes obtained from animals exposed to higher dose rates. A similar effect was observed in in vitro studies on V79 cells exposed to gamma radiation at 0.1-40 cGy/min dose rates [17]. The results show a significant decrease in the yield of $\gamma-\mathrm{H} 2 \mathrm{AX}$ foci in irradiated cells following reduction of radiation dose rate. The authors suggest that lower number of $\gamma$-H2AX foci in cells exposed to lower dose rates may be due to reduced probability of interactions between strand lesions and repair proteins.

However, in contrast to the results reported above our own research demonstrated that in the case of 400 cGy dose a lower dose rate (100 cGy/min) induced more micronuclei and apoptoses in human melanoma (Sk-mel), lung adenocarcinoma (A549), colon cancer (HCT), and normal bronchial epithelial cells (BEAS-2B), as compared to a higher dose rate $(600 \mathrm{cGy} / \mathrm{min})[18,19]$. The dose rate effect was not observed for cells lying outside irradiation area and exposed to scattered radiation dose. Our results were confirmed by a recent study in which comparison was made between low dose rate effect $(0.305$ cGy/min, ${ }^{125}$ J source) with that of a high dose rate (50 cGy/ min, ${ }^{60}$ Co source) upon neoplastic cells (A549 and H1299 lung cancer) as well as normal BEAS-2B cells, all exposed to 200-800 cGy doses [20]. These authors found that decreased survival and inhibition of cell cycle in G1/M phase was greater following exposure of cells to lower dose rate. Similarly, lower dose rate was also more effective in inducing apoptosis in cancer cells. Such an impact of dose rate was seen for doses exceeding 200 cGy [20]. Yet another study, performed on TK6 human lymphoblasts exposed to increased or decreased dose rates (0.22-10.6 cGy/min) demonstrated that the decreased dose rates were more effective in inducing micronuclei formation [21]. These researchers interpreted their results as the effect of interplay between DNA damage recognition and repair systems under changing dose rate conditions. Exposure to high dose rates initially triggers systems recognising DNA damage and starts repair processes but just as the dose rate gets smaller a decreased level is reached at which the damagerecognising systems become inactive. Under such conditions, unrepaired DNA damages accumulates and is seen as micronuclei. Conversely, when dose rates increase, the mechanisms recognizing and repairing DNA damage may be switched on with a delay but are functional throughout radiation exposure and do not lead to accumulation of greater numbers of micronuclei.

Earlier studies dealing with the inverse relationship between radiation dose rate magnitude and greater biological effectiveness ascribed more effective cell killing by lower dose rates to cell division block at the most radiation-sensitive G2/M phase of the cycle and accumulation of cells at this phase [22]. It was demonstrated later, however, that increased cell cycle blockage not always results in greater cell mortality and that an inverse dose rate effect occurs without cell cycle inhibition [23].

\section{Effect of dose rate on gene expression}

Another explanation of the inverse dose rate phenomenon was offered in a report from a study of gene expression in cells subjected to the same radiation dose but delivered at different dose rates [24]. The results suggest that, depending on dose rate magnitude, it is the expression of genes regulating apoptosis that becomes altered in lieu of genes controlling cell division. This is supposed to confirm the finding that dose rates can variably stimulate intercellular signaling responsible for apoptosis activation. An interesting result was obtained in experiments comparing biological effects induced in human glioma U87 cells using conventional iradiation (200 cGy/min dose rate) and Cyberknife-driven irradiation (400 cGy/min) [10]. The treatment with greater dose rates resulted in significantly activated TGF- $\beta$ and $\beta 1$-integrin pathways which mediate migration, adhesion and cell survival. These effects were not observed in cells exposed to conventional irradiation with the lower dose rate. The results suggest that greater dose rate can result in promotion of invasiveness of cancer cells. Also in vivo studies suggest that increasing dose rate of irradiation can result in activation of pathways which promote survival and progression of cancer cells. Gene expression profile was studied using experimental thymic lymphoma tumour-burdened mice irradiated from $\gamma-\left({ }^{137} \mathrm{Cs}\right)$ source with a 450 cGy dose, at 11.67 cGy/min or 80 cGy/ min dose rates [25]. Greater dose rates sped up tumour progression and precipitated death of animals. Irrespective of dose rates used, the radiation exposure enhanced expression of several genes linked to apoptosis as well as that of genes responsible for activation of $T$ and $B$ lymphocytes and proinflammatory cytokines. On the other hand, Jag2 gene linked to anticancer immune response, 
as well as the gene encoding IL-15 cytokine (which activates T lymphocytes and NK cells), were stimulated only in cells from mice exposed to low dose rate radiation. This strong immune response triggered by low dose rates could contribute to more effective elimination of cancer cells because mice irradiated under such conditions showed inhibited tumour development and increased survival. In mice irradiated with high dose rates a drastic lowering was noted in the expression of Sp3 gene inducing apoptosis, in genes stimulating T and B lymphocytes and in Traf6 gene regulating immune response following detection of cancer cells presence. This weakened immune response in mice exposed to irradiation with high dose rates could contribute to accelerated tumour progression and shortened survival [25].

\section{Summary}

The intention effect of using ionising radiation to eliminate cancer cells is to trigger lethal effects whereas damage to normal cells is collateral. Modern techniques used in oncological radiotherapy decrease patients' exposure time as a result of applying increased dose rates. Current conformal techniques based on three-dimensional treatment planning (3D-CRT) and modulation of radiation beam (IMRT, VMAT) allow safe application of high radiation doses to the tumour site while keeping surrounding tissues below irradiation levels considered to trigger complications. With these techniques exposure area is spatially and temporally regulated by modifying dose rate and beam shape. These new techniques considerably decrease irradiation time as compared to conventional techniques but, as demonstrated by molecular studies, dose rate alters gene expression profile for many important pathways responsible for tumour progression and immune response of the body.

Several reported studies have pointed to a definite trend of increased cell mortality in line with increased dose rates at a constant radiation dose $[8,10,11,16]$. This is not the rule, however. Other studies have demonstrated greater effectiveness of lower dose rates [18-21, 25]. To fully clarify this ambiguity, it is necessary to perform additional studies examining in depth signaling pathways in cells irradiated with dose rates commonly used in cancer radiotherapy. The anticipated results should verify whether the trend of using ever increasing dose rates does not, actually, leads to decreased effectiveness of radiotherapy.

The authors declare no conflict of interest.

\section{References}

1. Hall EJ, Brenner DJ. The dose-rate effect revisited: radiobiological considerations of importance in radiotherapy. Int J Radiat Oncol Biol Phys 1991; 21: 1403-14.

2. Widet M, Przybyszewski WM. Inverse dose-rate effect for the induction of micronuclei in Levis Lung Carcinoma after exposure to cobalt-60 rays. Radiat Res 1998; 14: 98-102.

3. Mitchell CR, Folkard M, Joiner MC. Effect of exposure to low-doserate ${ }^{60} \mathrm{Co}$ gamma rays on human tumour cells in vitro. Radiat Res 2002; 158: 311-8.
4. Sgouros G, Knox SJ, Joiner MC, Morgan WF, Kassis Al. MIRD continuing education: Bystander and low dose-rate effects: are these relevant to radionuclide therapy? J Nucl Med 2007; 48: 1683-91.

5. Przybyszewski WM, Wideł M, Szurko A, Maniakowski Z. Wpływ mocy dawki na komórkowe, biochemiczne i molekularne efekty promieniowania jonizującego. Postepy Hig Med Dosw 2008; 62: 468-77.

6. Chen H, Bao Y, Yu L, Jia R, Cheng W, Shao C. Comparison of cellular damage response to low-dose-rate 125 I seed irradiation and high-dose-rate gamma irradiation in human lung cancer cells. Brachytherapy 2012; 11: 149-56.

7. Hieber L, Ponsel G, Roos H, Fenn S, Fromke E, Kellever AM. Absence of a dose-rate effect in the transformation of C3H 10T1/2 cells by alpha particles. Int J Radiat Biol Relat Stud Phys Chem Med 1987; 52: 859-69.

8. Keall P, Chang M, Benedict S, Thames H, Vedam SS, Lin PS. Investigating the temporal effects on respiratory-gated and intensity-modulated radiotherapy treatment delivery on in vitro survival: an experimental and theoretical study. Int J Radiat Oncol Biol Phys 2008; 71: 1547-52.

9. Moiseenko V, Banáth JP, Duzenli C, Olive PL. Effect of prolonging radiation delivery time on retention of gammaH2AX. Radiat Oncol 2008; 3: 18.

10. Canazza A, De Grazia U, Fumagalli L, et al. In vitro effect of Cyberknife-driven intermittent irradiation on glioblastoma cell lines. Neurol Sci 2011; 32: 579-88.

11. Lohse I, Lang S, Hrbacek J, et al. Effect of high dose per pulse flattening filter-free beams on cancer cell survival. Radiother Oncol 2011; 101: 226-32.

12. Sorensen BS, Vestergaard A, Overgaard J, Praestegaard LM. Dependence of cell survival on instantaneous dose rate of a linear accelerator. Radiother Oncol 2011; 101: 223-25.

13. Verbakel WF, van den Berg J, Slotman BJ, Sminia P. Comparable cell survival between high dose rate flattening filter free and conventional dose rate irradiation. Acta Oncol 2013; 52: 652-7.

14. Valdiglesias V, Giunta S, Fenech M, Neri M, Bonassi S. $\gamma \mathrm{H} 2 \mathrm{AX}$ as a marker of DNA double strand breaks and genomic instability in human population studies. Mutat Res 2013; 753: 24-40.

15. Vral A, Fenech M, Thierens H. The micronucleus assay as a biological dosimeter of in vivo ionising radiation exposure. Mutagenesis 2011; 26: 11-17.

16. Turner HC, Shuryak I, Taveras M, et al. Effect of dose rate on residual $\gamma$-H2AX levels and frequency of micronuclei in $X$-irradiated mouse lymphocytes. Radiat Res 2015; 183: 315-24.

17. Kotenko KV, Bushmanov AY, Ozerov IV, et al. Changes in the number of double-strand DNA breaks in chinese Hamster V79 cells exposed to gamma-radiation with different dose rates. Int I Mol Sci 2013; 14: 13719-26.

18. Ślosarek K, Konopacka M, Rogoliński J, Latocha M, Sochanik A. Effect of depth on radiation-induced cell damage in a water phantom. Rep Pract Oncol Radiother 2005; 10: 37-41.

19. Ślosarek K, Konopacka M, Rogoliński J, Sochanik A. Effect of doserate and irradiation geometry on the biological response of normal cells and cancer cells under radiotherapeutic conditions. Mutat Res Genet Toxicol Environ Mutagen 2014; 773: 14-22.

20. Wang Z, Zhao Z, Lu J, Chen Z, Mao A, Teng G, Liu F. A comparison of the biological effect of ${ }^{125} \mathrm{~J}$ seeds continuous low-dose-rate radiation and ${ }^{60} \mathrm{Co}$ high-dose-rate gamma radiation on non-small cell lung cancer cells. PLoS One 2015; 10 (8): e0133728.

21. Brehwens K, Bajinskis A, Haghdoost S, Wójcik A. Micronucleus frequencies and clonogenic cell survival in TK6 cells exposed to changing dose rates under controlled temperature conditions. Int J Radiat Biol 2014; 90: 241-7.

22. Knox SJ, Sutherland W, Goris ML. Correlation of tumour sensitivity to low-dose-rate irradiation with G2/M-phase block and other radiobiological parameters. Radiat Res 1993; 135: 24-31. Erratum in: Radiat Res 1993; 136: 439.

23. DeWeese TL, Shipman JM, Dillehay LE, Nelson WG. Sensitivity of human prostatic carcinoma cells lines to low dose rate radiation exposure. J Urol 1998; 159: 591-8.

24. Amundson SA, Lee RA, Koch-Paiz CA, Bittner ML, Meltzer P, Trent $J M$, Fornace AJ Jr. Differential responses of stress genes to low dose-rate gamma irradiation. Mol Cancer Res 2003; 6: 445-52. 
25. Shin SC, Lee KM, Kang YM, et al. Differential expression of immune-associated cancer regulatory genes in low- versus highdose-rate irradiated AKR/J mice. Genomics 2011; 97: 358-63.

\section{Address for correspondence}

\section{Jacek Rogoliński}

Center for Translational Research and Molecular Biology of Cancer

Maria Skłodowska-Curie Memorial Cancer Center

and Institute of Oncology Gliwice Branch

Wybrzeże Armii Krajowej 15

44-101 Gliwice, Poland

e-mail: Jacek.Rogolinski@io.gliwice.pl

Submitted: 2.03 .2016

Accepted: $\quad 30.09 .2016$ 